\title{
Chromospheric heating and generation of plasma outflows by impulsively generated two-fluid magnetoacoustic waves
}

\author{
R. Niedziela ${ }^{1}$, K. Murawski ${ }^{1}$, and S. Poedts ${ }^{2,1}$ \\ ${ }^{1}$ Institute of Physics, University of M. Curie-Skłodowska, Pl. M. Curie-Skłodowskiej 1, 20-031 Lublin, Poland \\ ${ }^{2}$ Centre for Mathematical Plasma Astrophysics / Department of Mathematics, KU Leuven, Celestijnenlaan 200B, 3001 Leuven, \\ Belgium
}

June 15,2021

\begin{abstract}
Context. The origin of the heating of the solar atmosphere is still an unsolved problem. As the photosphere and chromosphere radiate more energy than the solar corona it is challenging but important to reveal all the mechanisms that contribute to plasma heating there. Ion-neutral collisions could play an important role.

Aims. Impulsively generated two-fluid magnetoacoustic waves are investigated in the partially ionized solar chromosphere and the associated heating and plasma outflows are studied, which higher up may result in nascent solar wind.

Methods. To describe the plasma dynamics, a two-fluid model is applied in which ions+electrons and neutrals are treated as separate fluids. The two-fluid equations are solved numerically using the JOANNA code.

Results. We show that magnetoacoustic waves, triggered in the photosphere by localised velocity pulses, can steepen into shocks which heat the chromosphere through ion-neutral collisions. Larger amplitude and wider pulses more effectively heat plasma and generate larger plasma outflows. Rising the altitude at which the pulse is launched, results in opposite effects, mainly in local cooling of the chromosphere, and slower plasma outflows.

Conclusions. Even a solitary pulse results in a train of waves. These waves can transform to shock waves and release thermal energy, heating the chromosphere significantly. A pulse can drive vertical flows which higher up can result in the origin of the solar wind.
\end{abstract}

Key words. Sun: activity - Sun: chromosphere

\section{Introduction}

One of the main problems of heliophysics concerns the source of the thermal energy required to heat the solar atmosphere of which the lowest, about $500 \mathrm{~km}$ thick layer is called the photosphere. The chromosphere, which is about $1.5 \mathrm{Mm}$ thick, caps this layer. The narrow transition region separates the chromosphere from the solar corona above it. Temperatures in the photosphere vary from about $5600 \mathrm{~K}$ at its bottom to about $4300 \mathrm{~K}$ at its top. In the chromosphere the temperature rises to about $10^{4} \mathrm{~K}$ in the middle chromosphere $( \pm 800- \pm 1800 \mathrm{~km}$ above the solar surface). Due to the temperatures in the photosphere and low and middle chromosphere, the plasma in these layers is only partially ionized. Recombination, photo-ionization and chemical ionization was discussed by Carlsson \& Stein (2002). At the top of the photosphere, the ionization degree is as low as $10^{-4}$, so there are about $10^{4}$ neutrals per ion. In the chromosphere, the relative number of ions to neutrals grows with height, i.e. temperature (Khomenko et al. 2014a). As a result of its $1-3 \mathrm{MK}$ average temperature, the plasma in the corona is essentially fully ionized.

The plasma density is also higher in the chromosphere and thus it radiates more than the corona. Hence, an additional mechanism of heating is required to compensate for these radiative losses (Narain \& Ulmschneider 1996). One of the sources of this heating may be associated with collisions between particles, which lead to wave damping (Erdélyi \& James 2004). The idea of heating the solar chromosphere by acoustic waves is very old and was first developed by Biermann (1946) and Schwarzschild (1948), who suggested that acoustic waves play a major role in the heating of this atmospheric layer. Later, the problem was widely studied by, e.g., Carlsson \& Stein (1995), Ulmschneider \& Musielak (2003) and Kuźma et al. (2019). The authors of the latter paper focused on the acoustic waves with wave periods within the range $30-200 \mathrm{~s}$ and showed that these waves are able to heat the chromosphere. The idea of heating the solar atmosphere without shock waves was studied by Wójcik et al. (2020a) who showed that the thermal energy release ratio during the ion-neutral collisions is sufficient to balance the radiative and thermal energy losses. Recently, Kuźma et al. (2021) focused on the studies of ion magnetoacoustic and neutral acoustic waves in a magnetic arcade and found that in the chromosphere the wave characteristics strongly depend on the magnetic field configuration. The advantages of using the two-fluid model over the single-fluid model have been studied by Zaqarashvili et al. (2011b). The single-fluid (MHD) model is sufficiently accurate for slow processes but fails on time-scales shorter than the ion-neutral collision time. Hence, the two-fluid approximation should be used for studying faster processes.

The problem of atmospheric heating was also studied in the context of Alfvén waves (Piddington 1956; Osterbrock 1961). Mechanism of damping of these waves by ion-neutral collision were studied by De Pontieu et al. (2001). In the lower chromosphere, collisional effects were found to be the most im- 
portant component of the dissipation mechanism (Khodachenko et al. 2004). As a matter of fact, Alfvén waves can dissipate their energy in the chromosphere as a result of ion-neutral collisions (Leake et al. 2005; Goodman 2011; Tu \& Song 2013; Zaqarashvili et al. 2013; Arber et al. 2016; Shelyag et al. 2016; Soler et al. 2017). This mechanism is, however, effective only in the chromosphere and thus not in the photosphere nor in the corona due to weak magnetization and strong ionization, respectively. In other studies, Zaqarashvili et al. (2013) and Soler et al. (2017) focused on the dissipation of torsional Alfvén waves and showed that they can by effectively dissipated in the chromosphere.

The present mechanisms considered for solar coronal heating are essentially based on magnetic field reconnection and resonant dissipation and/or phase-mixing of magnetohydrodynamic (MHD) waves. The later mechanism was reviewed recently by Van Doorsselaere et al. (2020). who showed that various wave modes may contribute to the heating of the solar corona. The effect of the cooling/heating on the dynamics of the MHD waves was investigated by Nakariakov et al. (2017) who concluded that the perturbations of the thermal equilibrium, by e.g. slow magnetoacoustic waves, lead to a heating/cooling misbalance which acts as an energy exchange mechanism. For more recent studies of thermal misbalance, see also Prasad et al. (2021) who studied the damping of slow magnetosonic waves based on a new dispersion relation. The problem of damping slow magnetosonic waves in the solar corona was also investigated by Duckenfield et al. (2021), considering non-zero $\beta$ plasma, and Kolotkov et al. (2019) who presented three potential regimes of wave evolution depending upon characteristic timescales of the thermal misbalance. Recently, Kolotkov et al. (2020) constrained the coronal heating function by observations of slow magnetoacoustic waves.

The solar corona extends smoothly into the solar wind. Studies of the solar wind were initiated by Biermann (1951) and Parker (1965). Parker (1958) postulated that the interplanetary space is not void but that it is filled by the solar wind. An early model of the solar wind with the power spectrum of Alfvén and kink waves was developed by Tu (1987). Later on, a lot of attention was paid to study the formation of the solar wind by plasma outflows. For instance, Tu et al. (2005) found these outflows in coronal funnels at altitudes between 5 and $20 \mathrm{Mm}$ above the photosphere. Later, the problem was reconsidered by Wójcik et al. (2019) who proposed that jets and associated plasma outflows, generated by the solar granulation, may higher up result in the formation of the solar wind. Moving upwardly, the plasma requires a source of momentum which can be provided by MHD waves (Ofman 2005; Marsch 2006; De Pontieu et al. 2007). Alfvén waves, for instance, may drive plasma outflows (Hollweg et al. 1982; Kudoh \& Shibata 1999; Matsumoto \& Suzuki 2012). A model for coronal heating and solar wind acceleration by low-frequency Alfvén wave turbulence was presented by van der Holst et al. (2014).

The goal of the present paper is to focus on magnetoacoustic waves in the context of heating the chromosphere and the associated plasma outflows, and thus leave the role of Alfvén waves for future studies. The novelty of this work is that we here consider impulsively generated two-fluid magnetoacoustic waves in the context of solar chromospheric heating and plasma outflows. This paper is organized as follows. In Sec. 2, we present the twofluid equations that are considered for describing the dynamics of the partially ionized solar atmosphere. Numerical results are presented in Sec. 3. Section 4 concludes this paper by summarising the main results and briefly discussing them.

\section{Two-fluid numerical model of the partially ionized solar atmosphere}

\subsection{Two-fluid equations}

To describe the low layers of the solar atmosphere we use twofluid equations for ions + electrons $\left({ }_{i, e}\right)$ and neutrals $\left(_{n}\right)$ treated as separate interacting fluids (Zaqarashvili et al. 2011a; Leake et al. 2014; Oliver et al. 2016; Maneva et al. 2017; Popescu Braileanu et al. 2019):

$$
\begin{array}{r}
\frac{\partial \varrho_{\mathrm{i}}}{\partial t}+\nabla \cdot\left(\varrho_{\mathrm{i}} \mathbf{V}_{\mathrm{i}}\right)=0, \\
\frac{\partial \varrho_{\mathrm{n}}}{\partial t}+\nabla \cdot\left(\varrho_{\mathrm{n}} \mathbf{V}_{\mathrm{n}}\right)=0, \\
\frac{\partial\left(\varrho_{\mathrm{i}} \mathbf{V}_{\mathrm{i}}\right)}{\partial t}+\nabla \cdot\left(\varrho_{\mathrm{i}} \mathbf{V}_{\mathrm{i}} \mathbf{V}_{\mathrm{i}}+p_{\mathrm{ie}} \mathbf{I}\right)= \\
\varrho_{\mathrm{i}} \mathbf{g}+\frac{1}{\mu}(\nabla \times \mathbf{B}) \times \mathbf{B}+\mathbf{S}_{\mathrm{m}}, \\
\frac{\partial\left(\varrho_{\mathrm{n}} \mathbf{V}_{\mathrm{n}}\right)}{\partial t}+\nabla \cdot\left(\varrho_{\mathrm{n}} \mathbf{V}_{\mathrm{n}} \mathbf{V}_{\mathrm{n}}+p_{\mathrm{n}} \mathbf{I}\right)=\varrho_{\mathrm{n}} \mathbf{g}-\mathbf{S}_{\mathrm{m}}, \\
\frac{\partial E_{\mathrm{i}}}{\partial t}+\nabla \cdot\left[\left(E_{\mathrm{i}}+p_{\mathrm{ie}}+\frac{\mathbf{B}^{2}}{2 \mu}\right) \mathbf{V}_{\mathrm{i}}-\frac{\mathbf{B}}{\mu}\left(\mathbf{V}_{\mathrm{i}} \cdot \mathbf{B}\right)\right]= \\
\frac{\partial E_{\mathrm{n}}}{\partial t}+\nabla \cdot\left[\left(E_{\mathrm{n}}+p_{\mathrm{n}}\right) \mathbf{V}_{\mathrm{n}}\right]=\left(\varrho_{\mathrm{n}} \mathbf{g}-\mathbf{S}_{\mathrm{m}}\right) \cdot \mathbf{V}_{\mathrm{n}}+Q_{\mathrm{n}}, \\
\frac{\partial \mathbf{B}}{\partial t}=\nabla \times\left(\mathbf{V}_{\mathrm{i}} \times \mathbf{B}\right), \quad \nabla \cdot \mathbf{B}=0, \\
E_{\mathrm{i}}=\frac{\varrho_{\mathrm{i}} \mathbf{V}_{\mathrm{i}}^{2}}{2}+\frac{p_{\mathrm{ie}}}{\gamma-1}+\frac{\mathbf{B}^{2}}{2 \mu}, \quad E_{\mathrm{n}}=\frac{\varrho_{\mathrm{n}} \mathbf{V}_{\mathrm{n}}^{2}}{2}+\frac{p_{\mathrm{n}}}{\gamma-1} .
\end{array}
$$

Here, $\mathbf{I}$ is the identity matrix, $p_{i e, n}$ denote the ion + electron and neutral gas pressures, while $\varrho_{i, n}$ denote ion and neutral mass densities, and $\mathbf{V}_{i, n}$ the ion and neutral velocities. moreover, $\mathbf{g}=[0,-g, 0]$ is gravitational acceleration with its magnitude $g=274.78 \mathrm{~m} \mathrm{~s}^{-2}, \mathbf{B}$ is the magnetic field pointing in the $y$-direction, and $\mu$ the magnetic permeability of the medium. In Eqs. (3) - (6), $\mathbf{S}_{m}$ and $Q_{i, n}$ correspond to the collisional momentum and energy exchange terms, respectively. They are given as (Meier \& Shumlak 2012; Oliver et al. 2016)

$$
\begin{aligned}
\mathbf{S}_{\mathrm{m}} & =\varrho_{i} v_{\mathrm{in}}\left(\mathbf{V}_{\mathrm{n}}-\mathbf{V}_{\mathrm{i}}\right), \\
Q_{\mathrm{i}} & =\frac{1}{2} v_{\mathrm{in}} \varrho_{\mathrm{i}}\left(\mathbf{V}_{\mathrm{i}}-\mathbf{V}_{\mathrm{n}}\right)^{2}+\frac{k_{\mathrm{B}} v_{\mathrm{in}} \varrho_{\mathrm{i}}}{(\gamma-1) m_{\mathrm{n}}}\left(T_{\mathrm{n}}-T_{\mathrm{i}}\right), \\
Q_{\mathrm{n}} & =\frac{1}{2} v_{\mathrm{in}} \varrho_{\mathrm{i}}\left(\mathbf{V}_{\mathrm{i}}-\mathbf{V}_{\mathrm{n}}\right)^{2}+\frac{k_{\mathrm{B}} v_{\mathrm{in}} \varrho_{\mathrm{i}}}{(\gamma-1) m_{\mathrm{n}}}\left(T_{\mathrm{i}}-T_{\mathrm{n}}\right) .
\end{aligned}
$$

The ion-neutral collision frequency, $v_{i n}$, is defined as (Braginskii 1965; Ballester et al. 2018)

$v_{\mathrm{in}}=\frac{4}{3} \frac{\sigma_{\mathrm{in}} \varrho_{\mathrm{n}}}{m_{\mathrm{H}}\left(\mu_{\mathrm{i}}+\mu_{\mathrm{n}}\right)} \sqrt{\frac{8 k_{\mathrm{B}}}{\pi m_{\mathrm{H}}}\left(\frac{T_{\mathrm{i}}}{\mu_{\mathrm{i}}}+\frac{T_{\mathrm{n}}}{\mu_{\mathrm{n}}}\right)}$.

In the above equation, $m_{H}$ is the hydrogen mass, $\sigma_{\text {in }}=1.4$. $10^{-15} \mathrm{~cm}^{2}$ is the collision cross-section (Vranjes \& Krstic 2013), $\mu_{\mathrm{i}}=0.58$ and $\mu_{\mathrm{n}}=1.21$ are the mean masses of, respectively, ions and neutrals, and $T_{i, n}$ are the temperatures specified by the ideal gas laws,

$p_{\mathrm{ie}}=\frac{k_{\mathrm{B}}}{m_{i}} \varrho_{\mathrm{i}} T_{\mathrm{i}}, \quad$ and $\quad p_{\mathrm{n}}=\frac{k_{\mathrm{B}}}{m_{n}} \varrho_{\mathrm{n}} T_{\mathrm{n}}$.

Here, $m_{\mathrm{i}}=m_{\mathrm{H}} \mu_{\mathrm{i}}$, and $m_{\mathrm{n}}=m_{\mathrm{H}} \mu_{\mathrm{n}}$, where $m_{H}$ corresponds to the mass of a hydrogen atom, $k_{B}$ is the Boltzmann constant, and 
$\gamma=1.4$ corresponds to the specific heats ratio. As the photospheric plasma contains bi-atomic molecules, we decided to consider $\gamma=1.4$ instead of $5 / 3$. The latter value is suitable for plasma that only consists of atoms. For simplicity reasons, we neglected thermal conduction as well as other non-adiabatic and non-ideal terms in the two-fluid equations. We should expect that thermal conduction will impact wave propagation by diffusing thermal energy into less localised and thus less intensive plasma heated regions.

The two-fluid equations do not very well describe the top of the convection zone and the photosphere which are included in the simulation domain but they accurately describe the chromosphere and the low corona, which are the focus of our simulation model. Problems with the two-fluid equations result from comparable values of characteristic gyro- and collision frequencies in the photosphere (Khomenko et al. 2014b, Fig. 1, top), while fluid models require that the collision frequencies are much higher than the gyro-frequencies.

\subsection{Magnetohydrostatic equilibrium}

As an initial condition we assume that the atmosphere is static $\left(\mathbf{V}_{i, n}=\mathbf{0}\right)$, and it is in hydrostatic equilibrium with the ion and neutral pressure gradients being balanced by the gravity forces on both species:

$-\nabla p_{i, n}+\varrho_{i, n} \mathbf{g}=\mathbf{0}$.

Using the ideal gas laws in Eqs. (13) and the $y$-components of Eqs. (14), we express the background gas pressures and mass densities as

$p_{i, n}(y)=p_{i, n 0} \exp \left(-\int_{y_{r}}^{y} \frac{d y}{\Lambda_{i, n}(y)}\right)$,

$\varrho_{i, n}(y)=\frac{p_{i, n}(y)}{g \Lambda_{i, n}(y)}$,

where $\Lambda_{i, n}=k_{B} T /\left(\mu_{i, n} g\right)$ are ion and neutral pressure scale heights, respectively, while $p_{i 0}=0.01 \mathrm{~Pa}$ and $p_{n 0}=0.0003 \mathrm{~Pa}$ denote the corresponding gas pressures at the reference level, taken here at $y=y_{r}=50 \mathrm{Mm}$.

We consider the height-dependent temperature, $T(y)$, model of Avrett \& Loeser (2008), see Fig. 1 (top). This model also determines the equilibrium mass densities and gas pressure profiles. As the electron mass is very small in comparison to the mass of neutrals and ions, we neglect the electron dynamics. Note that the ion mass density is lower than that of the neutral component in the photosphere and low chromosphere (Fig. 1, middle). However, above the altitude of $y \approx 0.9 \mathrm{Mm}$ and especially above the transition region, which is located at $y \approx 2.1 \mathrm{Mm}$, neutrals are much less numerous than ions. The presence of gravity in the system leads to the appearance of an acoustic cut-off period (Fig. 1, bottom). The formula is given as

$P_{\mathrm{ac}}=\frac{4 \pi \Lambda}{C_{S} \sqrt{1+2 \frac{d \Lambda}{d y}}}$,

where $\Lambda$ is the pressure scale height, and $C_{s}=$ $\sqrt{\gamma\left(p_{i}+p_{n}\right) /\left(\varrho_{i}+\varrho_{n}\right)}$ is the sound speed.

The pressure scale height $\Lambda$ is responsible for the propagating of the waves when their period $P$ is smaller than the $P_{\mathrm{ac}}$. Otherwise, if this value is greater than or equal to $P_{\mathrm{ac}}$, the waves are evanescent (Horace 1909). It is worth to notice that the here considered $P_{\mathrm{ac}}$ is a local quantity that varies with height
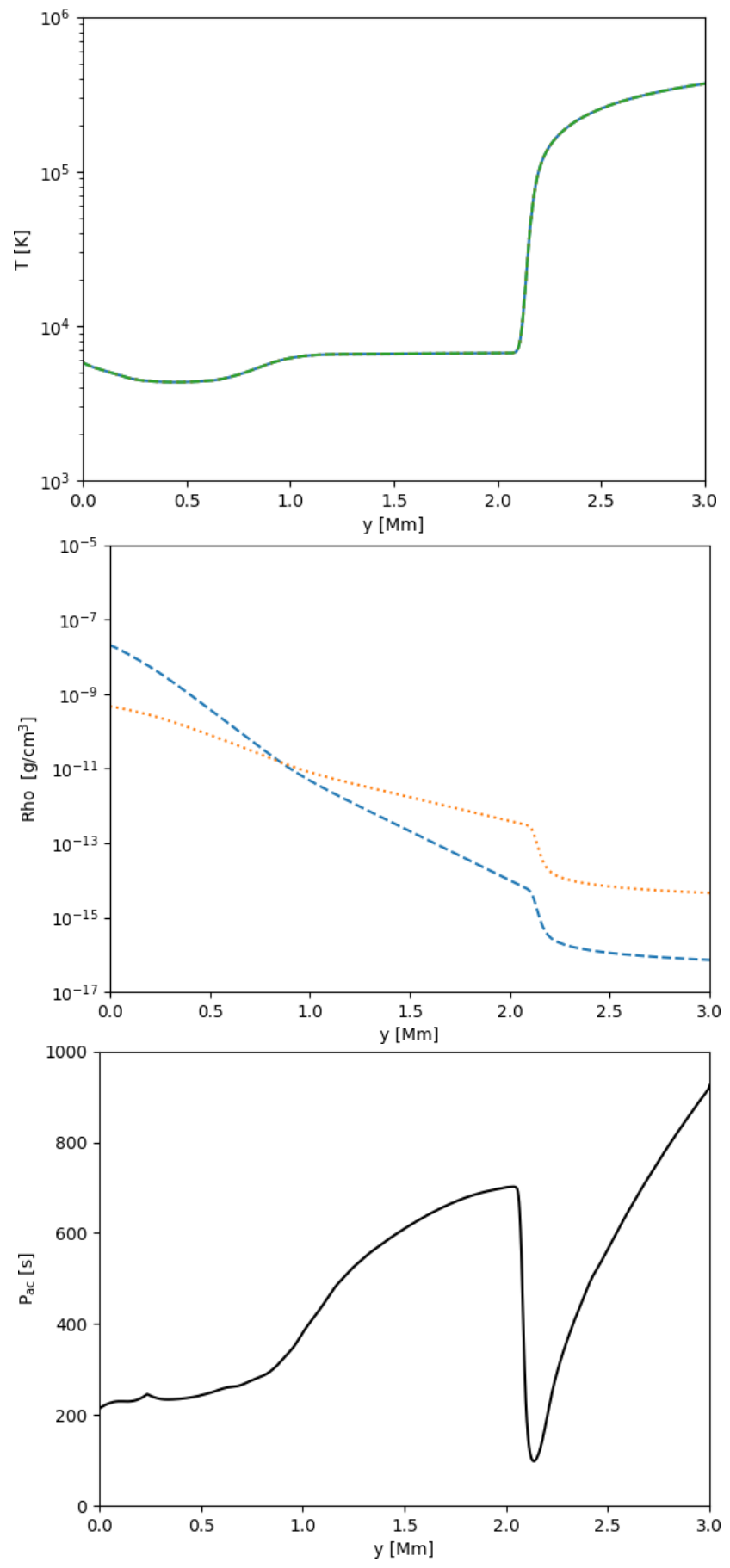

Fig. 1. Variation with height of the equilibrium temperature (top), mass densities (middle) of neutrals (dashed line) and ions (dotted line), and acoustic cut-off period (bottom).

(Musielak et al. 2006).

The above described hydrostatic equilibrium is overlaid by a uniform vertical magnetic field of magnitude $B_{y}=10$ Gs. Such a magnetic field is current-free $(\nabla \times \mathbf{B} / \mu=0)$ and hence, force-free $((\nabla \times \mathbf{B}) \times \mathbf{B} / \mu=0)$, and thus it does not alter the above described hydrostatic equilibrium. 


\subsection{Impulsive perturbations}

At $t=0 \mathrm{~s}$, when the numerical simulations are started, we perturb the magnetohydrostatic equilibrium by a localised signal in the vertical components of the ion and neutral velocities, viz.

$V_{\text {iy }}(y, t=0)=V_{n y}(y, t=0)=A \exp \left(-\left(\frac{y-y_{0}}{w}\right)^{2}\right)$,

where $A$ is the amplitude of the pulse, $w$ its width, and $y_{0}$ the height from which the pulse is launched. We consider the following cases: $A=0.5 \mathrm{~km} \mathrm{~s}^{-1}, A=1 \mathrm{~km} \mathrm{~s}^{-1}, A=2 \mathrm{~km} \mathrm{~s}^{-1}$, and $A=-5 \mathrm{~km} \mathrm{~s}^{-1}$, combined with different widths of the pulse, namely $w=0.1 \mathrm{Mm}, w=0.25 \mathrm{Mm}$, and $w=0.3 \mathrm{Mm}$; and various launching heights, viz. $y_{0}=0 \mathrm{Mm}, y_{0}=0.25 \mathrm{Mm}$, and $y_{0}=0.5 \mathrm{Mm}$. The first two values of the amplitude $A$ represent a typical flow associated with the solar granulation (e.g., Falco et al. 2017), while the third value corresponds to a stronger signal, and the last value mimics downdrafts. The implied maximum value of the pulse amplitude $A=0.5 \mathrm{~km} \mathrm{~s}^{-1}$ is about six times larger than the local Alfvén speed, which for the used value of $B_{y}=10 \mathrm{Gs}$ is about $C_{A}=0.08 \mathrm{~km} \mathrm{~s}^{-1}$ at $y=0 \mathrm{Mm}$ and much smaller than the local sound speed which is about $C_{S}=7.5 \mathrm{~km} \mathrm{~s}^{-1}$ at $y=0 \mathrm{Mm}$. The characteristic width of the pulse, $w=300 \mathrm{~km}$, is comparable to the size of the smallest granules. The difference between the positive and negative values of the amplitude results in the ion and neutral fluids being pushed upwards and downwards, respectively. But both negative and positive pulses spread into upward and downward propagating waves. Note that $y_{0}=0 \mathrm{Mm}$ corresponds to the bottom of the photosphere, $y_{0}=0.25 \mathrm{Mm}$ to the middle of the photosphere, and $y_{0}=0.5 \mathrm{Mm}$ is associated with its top. For simplicity reasons, we consider only pulses in the ion and neutral vertical velocity components. Obviously, the physical nature of realistic perturbations is more complex than the dependence presented by Eq. (18). Moreover, we consider an impulsive perturbation launched at $t=0 \mathrm{~s}$, without first relaxing the system, because the relative numerical errors are small in all atmospheric layers (Fig. 2).

\section{Numerical results}

The one-dimensional numerical simulations are performed with the JOANNA code (Wójcik et al. 2020b), which solves the above-mentioned two-fluid equations. We assume that the system has a slab geometry and that it is invariant along the $x$ and $z$-directions $(\partial / \partial x=\partial / \partial z=0)$, and the $z$-components of the velocities and the magnetic field are set identically zero $\left(V_{i z}=V_{n z}=B_{z}=0\right)$. Applying these simplifications, we remove Alfvén waves from the system which guides (affected by gravity) magnetoacoustic waves. Internal-gravity waves are absent in the system as they are unable to propagate along the gravity action.

The simulation domain is specified as $-0.5 \leq y \leq 60.0 \mathrm{Mm}$. The distance below $6.87 \mathrm{Mm}$ is divided into 1024 cells, thus the size of the grid cells is $\Delta y \approx 7.2 \mathrm{~km}$. Higher up, the grid is stretched and the remainder of the domain is covered by 128 cells. At the top and bottom boundaries of the simulation region, we set and hold fixed all plasma quantities to their equilibrium values, given by Eqs. (15) and (16).

\subsection{Numerical test}

Figure 2 illustrates time-distance plots for the ratio between the vertical component of the ion velocity and the local sound
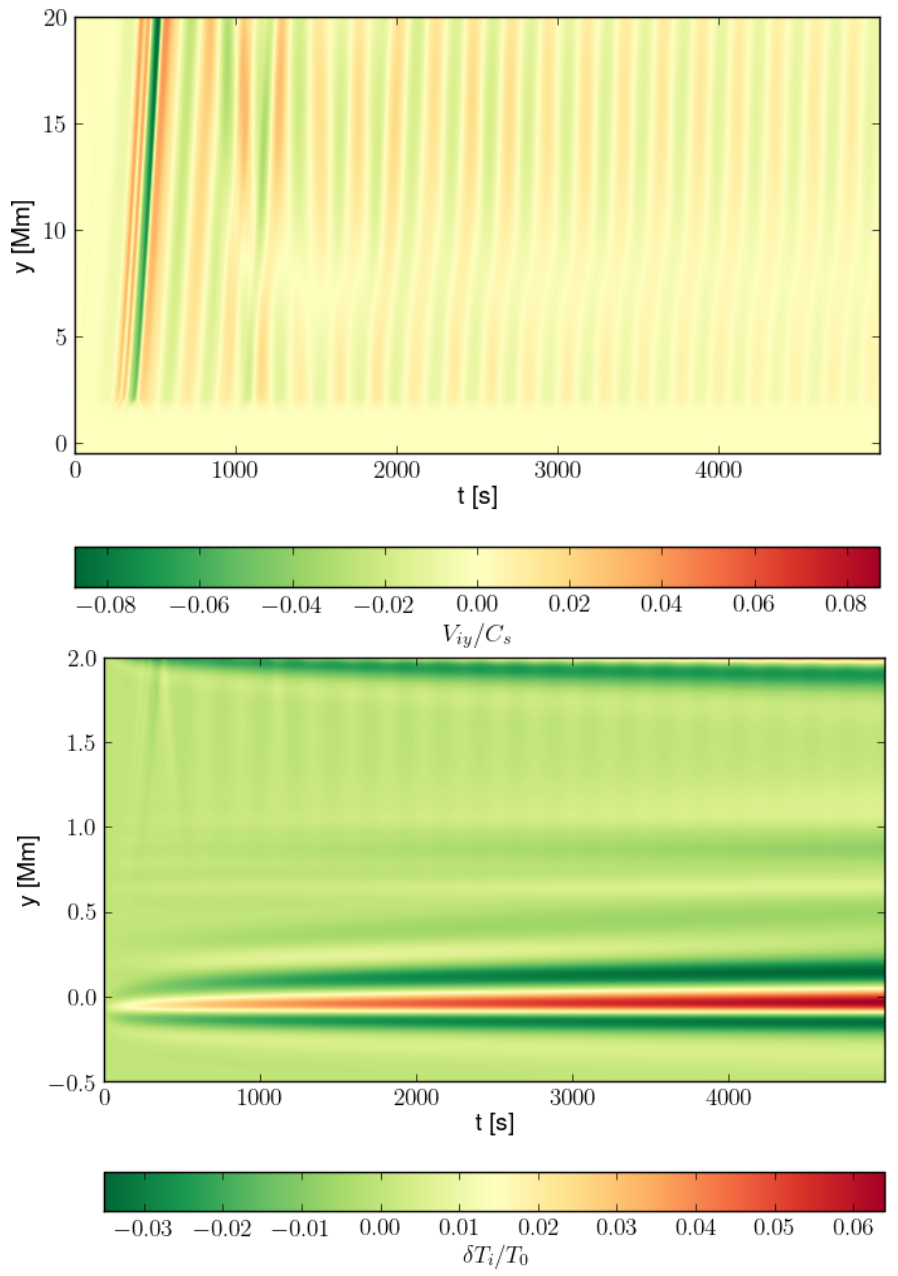

Fig. 2. Time-distance plots for $V_{\mathrm{iy}} / C_{s}$ (top) and $\delta T_{i} / T$ (bottom) in the case of no pulse $(A=0)$.

speed, $V_{\text {iy }} / C_{S}$, (top) and the relative perturbed temperature of ions, $\delta T_{i} / T=\left(T_{i}-T\right) / T$, (bottom) in the absence of any initial pulse (i.e. for $A=0$ in Eq. 18). The signals present in both panels result from numerical errors which are inherent features of every numerical code. As the $\max \left|V_{\text {iy }} / C_{s}\right| \approx 0.08$ occurs in the corona, and it indicates that the vertical velocities resulting from the discretization are small in comparison to the local sound speed, we infer that the numerical errors induced in the velocity component $V_{\text {iy }}$ are negligible. In the lower regions of the atmosphere, the numerical errors decrease to $\max \left|V_{\text {iy }} / C_{s}\right| \approx 0.02$ in the chromosphere, and $\max \left|V_{\mathrm{iy}} / C_{s}\right| \approx 0.0002$ in the photosphere. Similarly, $\max \left|\delta T_{i} / T\right| \approx 0.065$, showing that the numerical errors detected in the perturbed ion temperature are negligibly small too. In contrast to the numerical errors in velocity, the errors in the perturbed temperature decrease with height to $\max \left|\delta T_{i} / T\right| \approx 0.03$ in the chromosphere, and $\max \left|\delta T_{i} / T\right| \approx 0.02$ in the corona. The apparent periodicity in $\delta T_{i} / T$ (Fig. 2, bottom) may coincide with the acoustic cut-off period of the numerical noise.

\subsection{Magnetoacoustic waves}

\subsubsection{Pulse amplitude effects}

We first considered an initial pulse at the bottom of the photosphere $\left(y_{0}=0 \mathrm{Mm}\right)$. The initial pulse, given by Eq. (18), excites magnetoacoustic waves. These waves can get shocked in the chromosphere (Snow \& Hillier 2021). The formation of 

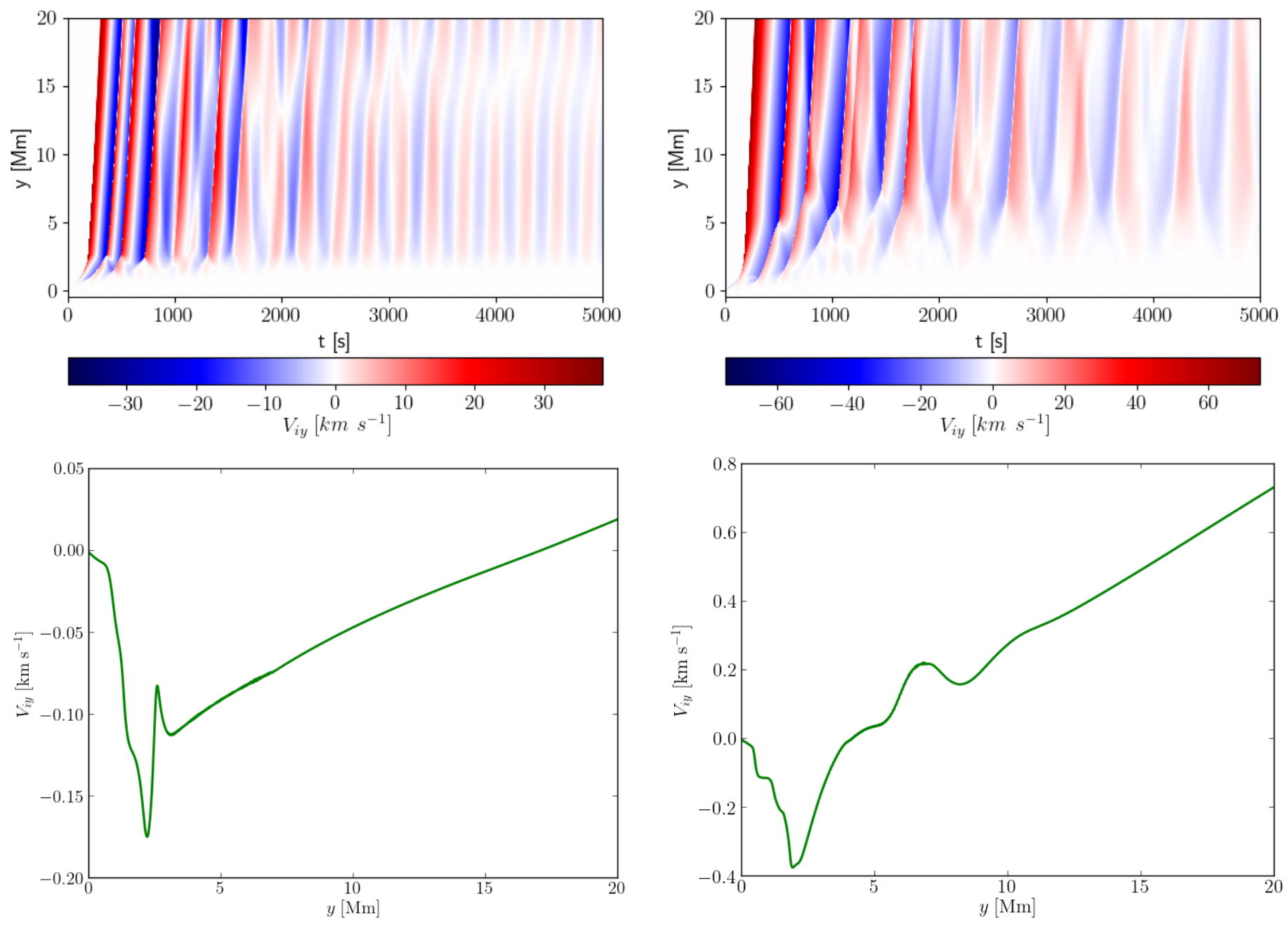

Fig. 3. Time-distance plots for $V_{\text {iy }}$ (top) and averaged $V_{\text {iy }}$ over time (bottom) for $y_{0}=0 \mathrm{Mm}, A=0.5 \mathrm{~km} \mathrm{~s}^{-1}$ (left), and $A=-5 \mathrm{~km} \mathrm{~s}{ }^{-1}$ (right). The velocity is expressed in units of $\mathrm{km} \mathrm{s}^{-1}$.

shock waves is clearly seen in the time-distance plots for $V_{\text {iy }}$ (Fig. 3, top), and it is stronger for a larger value of $|A|$ (righttop). These shocks heat plasma through ion-neutral collisions. Similarly to the gravity action, two-fluid effects introduce dispersion for the ion magnetoacoustic and neutral acoustic waves which are described by the Eqs. (1) - (13). As a result of this dispersion, the initial pulse spreads into a train of upward and downward propagating waves. The transition region oscillates as a natural consequence of the incoming train of waves which is well seen as upward and downward propagating waves. These waves move with the local tube speed which is sub-sonic and sub-Alfvénic. As gravity introduces characteristic spatial scales in the form of ion and neutral pressure scale heights, the amplitude of the oscillations in $V_{\text {iy }}$ grow with height (top panels). At the top of the photosphere, i.e. at $\pm 0.5 \mathrm{Mm}$, the signal in $V_{\text {iy }}$ is quite small and it reaches a maximum value there of $0.8 \mathrm{~km} \mathrm{~s}^{-1}$ for $A=0.5 \mathrm{~km} \mathrm{~s}^{-1}$ and $9 \mathrm{~km} \mathrm{~s}^{-1}$ for $A=-5 \mathrm{~km} \mathrm{~s}^{-1}$. Similarly at later times, after $t=10^{3} \mathrm{~s}$ the signal is very weak as the impulsively generated magnetoacoustic waves already left the system. Shock waves are generated up to $t \approx 700 \mathrm{~s}$. This means that due to this initial pulse, thermal energy is essentially released until this time since it is mainly generated at the shocks by ion-neutral collisions. The initial pulse also induces plasma flows. These are evident from the temporarily averaged vertical ion velocity given as

$\left\langle V_{\text {iy }}\right\rangle=\frac{1}{t_{2}-t_{1}} \int_{t_{1}}^{t_{2}} V_{\text {iy }} d t$,

where $t_{1}=0 \mathrm{~s}$ and $t_{2}=5000 \mathrm{~s}$. The outflows occur in the corona (Fig. 3, bottom). A higher amplitude of the pulse results in faster outflows originating from lower levels of the atmosphere. In particular, for $A=0.5 \mathrm{~km} \mathrm{~s}^{-1},\left\langle V_{\text {iy }}\right\rangle$ grows essentially linearly with height $y$, reaching a value of $0.02 \mathrm{~km} \mathrm{~s}^{-1}$ at $y=20 \mathrm{Mm}$ (Fig. 3, bottom-left). For $A=-5 \mathrm{~km} \mathrm{~s}^{-1}$ the growth of $\left\langle V_{\text {iy }}\right\rangle$ with $y$ is more abrupt than in the case of $A=0.5 \mathrm{~km} \mathrm{~s}^{-1}$ and $\left\langle V_{\text {iy }}\right\rangle$ attains a value of about $0.7 \mathrm{~km} \mathrm{~s}^{-1}$ at $20 \mathrm{Mm}$. This speed is smaller than in the semi-empirical data of Avrett \& Loeser (2008), where the velocity is of the order of $40 \mathrm{~km} \mathrm{~s}^{-1}$ at $y=3 \mathrm{Mm}$. In the lower atmospheric regions down-flows are observed. Such up-flows of $\left\langle V_{\mathrm{iy}}\right\rangle=2 \mathrm{~km} \mathrm{~s}^{-1}$ and down-flows of $\left\langle V_{\text {iy }}\right\rangle<10 \mathrm{~km} \mathrm{~s}^{-1}$ were reported by Kayshap et al. (2015) and more recently also by Tian et al. (2021).

Figure 4 (left panel) illustrates the ion mass flux, $F_{\varrho_{i y}}=$ $\varrho_{i y} V_{\text {iy }}$, which in contrast to the ion velocity, reaches its highest values below the transition region. Negative values of the flux are observed only in the initial phase of the wave evolution lasting up to $t \approx 200 \mathrm{~s}$. The magnetoacoustic waves, which are reflected from the transition region, are represented by the stripes leaning 

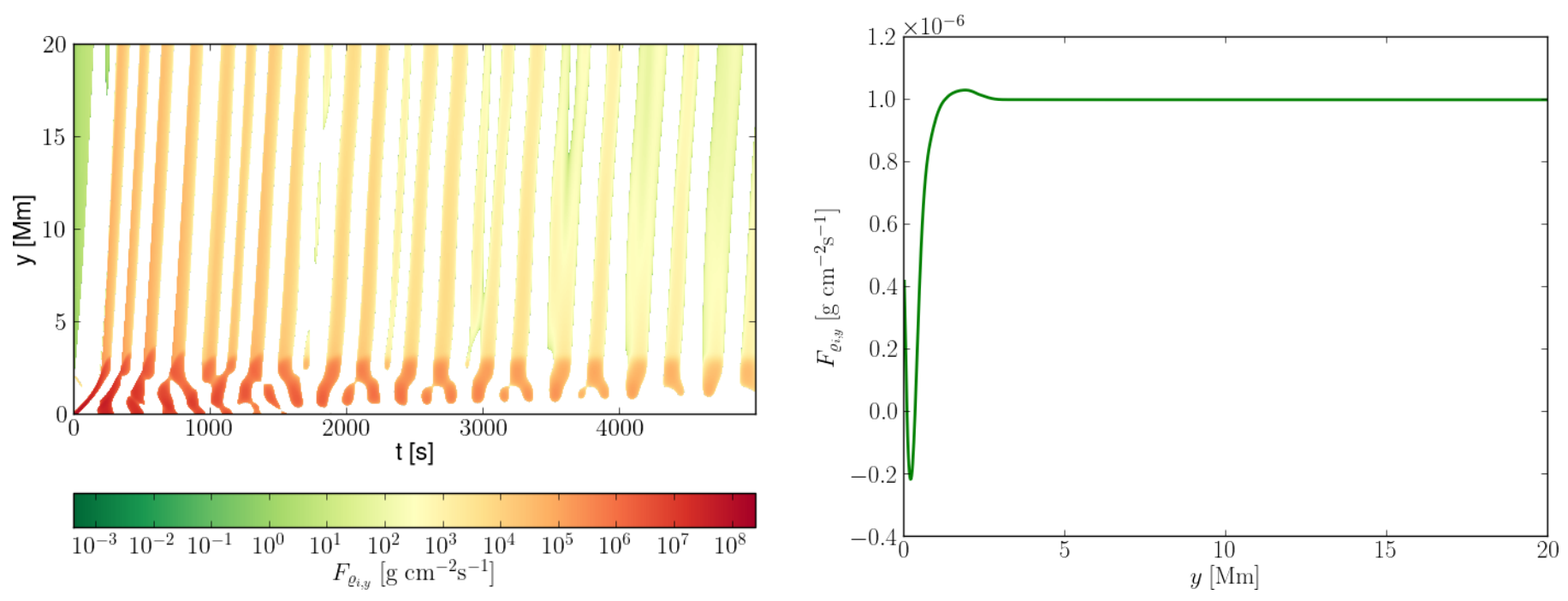

Fig. 4. Time-distance plots for ion mass flux, $F_{\varrho_{i, y}}=\varrho_{i} V_{\text {iy }}$ (left), and its averaged over time profile (right) for the initial pulse with width $w=0.3 \mathrm{Mm}$ and amplitude $A=0.5 \mathrm{~km} \mathrm{~s}^{-1}$, launched from $y=y_{0}=0 \mathrm{Mm}$.

towards the $y$-axis. The right panel presents the vertical profile of the averaged (over time) ion mass flux which reaches its lowest value of $F_{\varrho_{i, y}}=-0.2 \times 10^{-6} \mathrm{~g} \mathrm{~cm}^{-2} \mathrm{~s}^{-1}$ at $y=0.2 \mathrm{Mm}$. Higher up, it abruptly grows to $F_{\varrho_{i, y}}=10^{-6} \mathrm{~g} \mathrm{~cm}^{-2} \mathrm{~s}^{-1}$ at $y=1.5 \mathrm{Mm}$ and then remains essentially constant with height.

Figure 5 (top) illustrates the velocity drift, $\delta V=V_{\text {iy }}-V_{n y}$, which grows with height reaching its maximum magnitude in the upper chromosphere. However, at the bottom of the numerical region ions and neutrals are strongly coupled, thus they propagate with essentially the same velocity. As the quantity $\delta V$ attains its largest values in the initial phase, we infer that plasma heating can take place up to $t=10^{3} \mathrm{~s}$ after the initial pulse. The values of the velocity drift are small, which indicates that ions and neutrals are strongly coupled. In the right-top panel, at the bottom of the photosphere, $y=0 \mathrm{Mm}$, and below at $y \approx 0.2 \mathrm{Mm}$ and $y \approx 0.5 \mathrm{Mm}$ we observe some oscillatory signatures. The first two look like quasi-harmonic, decaying oscillations and the third one is seen as propagating upward and faster decaying waves. Since they appear at the bottom of the simulation box they may result from numerical errors.

The middle panels of Fig. 5 show the relative perturbed temperature specified as

$\frac{\delta T_{i}}{T}=\frac{T_{i}-T}{T}$.

This quantity reaches its largest magnitude of 0.55 at $y \approx 2 \mathrm{Mm}$ for $A=0.5 \mathrm{~km} \mathrm{~s}^{-1}$ and 0.8 at $y \approx 2 \mathrm{Mm}$ for $A=-5 \mathrm{~km} \mathrm{~s}^{-1}$, while the photosphere is hardly heated at all. Notice that the signals in the top and middle panels exhibit a level of correlation, which means that the chromospheric plasma is heated by the ionneutral collisions.

From Eqs. (10) and (11) we infer that the velocity drift, $\delta V$, contributes an important part to the heating which is particularly effective at shocks, which is clearly seen in Fig. 5 (top). This was also noticed by Zhang et al. (2020) and Snow \& Hillier (2021) after similar simulations in a different set up. This heating can be quantified by the quantity $\delta T_{i} / T$ which is displayed on Fig. 5 (middle).

We define the time averaged relative perturbed temperature as

$\left\langle\frac{\delta T_{i}}{T}\right\rangle=\frac{1}{t_{2}-t_{1}} \int_{t_{1}}^{t_{2}} \frac{\delta T_{i}}{T} d t$, where $t_{1}=0 \mathrm{~s}$ and $t_{2}=5000 \mathrm{~s}$. This quantity is illustrated in Fig. 5, bottom. Notice that the chromosphere is more strongly heated for higher values of the pulse amplitude $|A|$. The lowest averaged temperature is seen in the top of the photosphere and above this region the atmosphere is heated more. For $A=$ $-5 \mathrm{~km} \mathrm{~s}^{-1}$, all values of $\left\langle\delta T_{i} / T\right\rangle$ are positive and the minimum of $\left\langle\delta T_{i} / T\right\rangle \approx 0$ at $y \approx 0.4 \mathrm{Mm}$, takes place at the equilibrium temperature minimum (Avrett \& Loeser 2008), where the magnetoacoustic waves experience reflections, and reaching a value of about 2.3 at $y=2 \mathrm{Mm}$, at the top of the chromosphere. A characteristic flattening is present within the lower levels of the middle chromosphere, from $y \approx 0.8 \mathrm{Mm}$ to $y \approx 1 \mathrm{Mm}$. Above this plateau $\left\langle\delta T_{i} / T\right\rangle$ suddenly increases with $y$ (bottom-right).

Notice that the temperature is reduced permanently in the photosphere with essentially unseen waves in $\delta T_{i} / T$ (Fig. 5, middle). In the chromosphere, we observe oblique stripes which are leaned towards the vertical axis and which represent magnetoacoustic waves. In the case of $A=0.5 \mathrm{~km} \mathrm{~s}^{-1}$, max $\left(\delta T_{i} / T\right) \approx 0.6$ at $y=1.8 \mathrm{Mm}$, which is located in the chromosphere (middle-left). As for larger absolute values of amplitude, e.g. for $A=-5 \mathrm{~km} \mathrm{~s}^{-1}$ (middle-right), the generated shocks are more pronounced than for $A=0.5 \mathrm{~km} \mathrm{~s}^{-1}$, and they result in more intensive heating with $\max \left(\delta T_{i} / T\right) \approx 0.8$ in the middle of the chromosphere, at $y=1.1 \mathrm{Mm}$ (Fig. 5, middle).

\subsubsection{Pulse width effect}

In order to study how the width of the pulse, $w$, affects the plasma heating, we perform some parametric studies. From Fig. 6 (top) we infer that increasing $w$ from $0.1 \mathrm{Mm}$ to $0.25 \mathrm{Mm}$ results in a significant heating of the chromosphere. However, for $w=$ $0.1 \mathrm{Mm}$ only the top layer of the chromosphere is heated (topleft), while a wider pulse width, $w=0.3 \mathrm{Mm}$, results in heating of the whole chromosphere (top-right). Similarly, the cooling during the first $200 \mathrm{~s}$ is more pronounced for the wider pulse, and it results from the plasma being rarefied.

\subsubsection{Pulse launching height effect}

Figure 6 (bottom) shows time-distance plots for $\delta T_{i} / T$ for different pulse launching heights, i.e. for different values of the pa- 

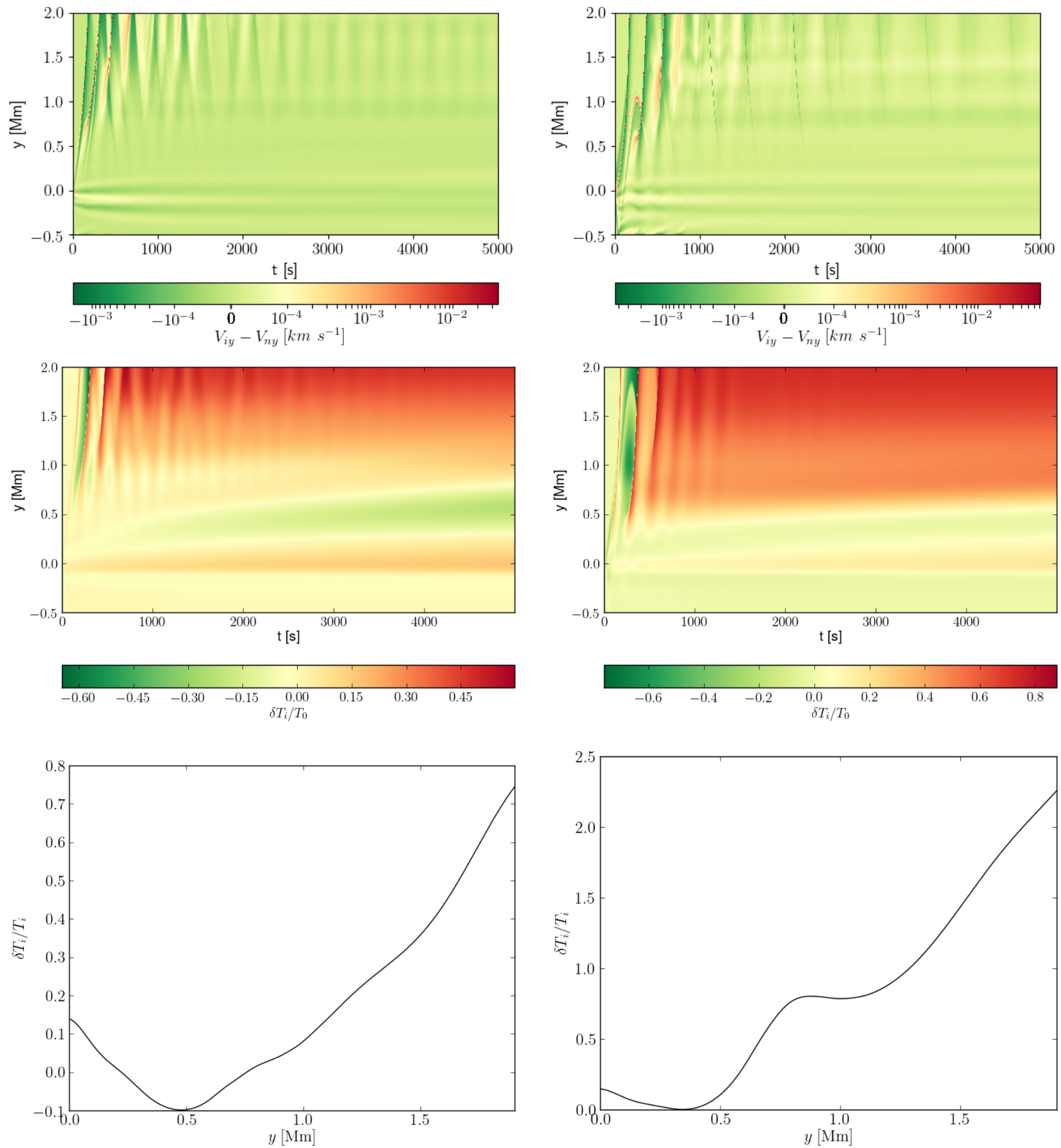

Fig. 5. Time-distance plots for the velocity drift, $V_{\mathrm{iy}}-V_{n y}$ (top), $\delta T_{i} / T$ (middle), and averaged $\delta T_{i} / T$ over time (bottom) for the pulse with width $w=0.3 \mathrm{Mm}$, launched from $y=y_{0}=0 \mathrm{Mm}$ and with amplitude: $A=0.5 \mathrm{~km} \mathrm{~s}^{-1}$ (left) and $A=-5 \mathrm{~km} \mathrm{~s}^{-1}$ (right).

rameter $y_{0}$. A higher value of $y_{0}$ results in a smaller value of $\max \left(\delta T_{i} / T\right)$ at a given height $y$. This is a consequence of a pressure scale-height over which, according to the linear theory, the amplitude of the signal grows $e$-times (e.g. Nakariakov \& Verwichte 2005). For the pulse launched from a higher level $y_{0}$, with the same amplitude, the signal passes fewer scale-heights reach- ing a given level $y$. Consequently, $\delta V$ and $\delta T_{i} / T$ are smaller (at a given height $y$ ) for a larger value of $y_{0}$.

Figure 7 illustrates the relative perturbed temperature averaged over time and height, $H$, defined as

$$
H=\frac{1}{y_{1}-y_{0}} \int_{y_{0}}^{y_{1}}\left\langle\frac{\delta T_{i}}{T}\right\rangle d y .
$$



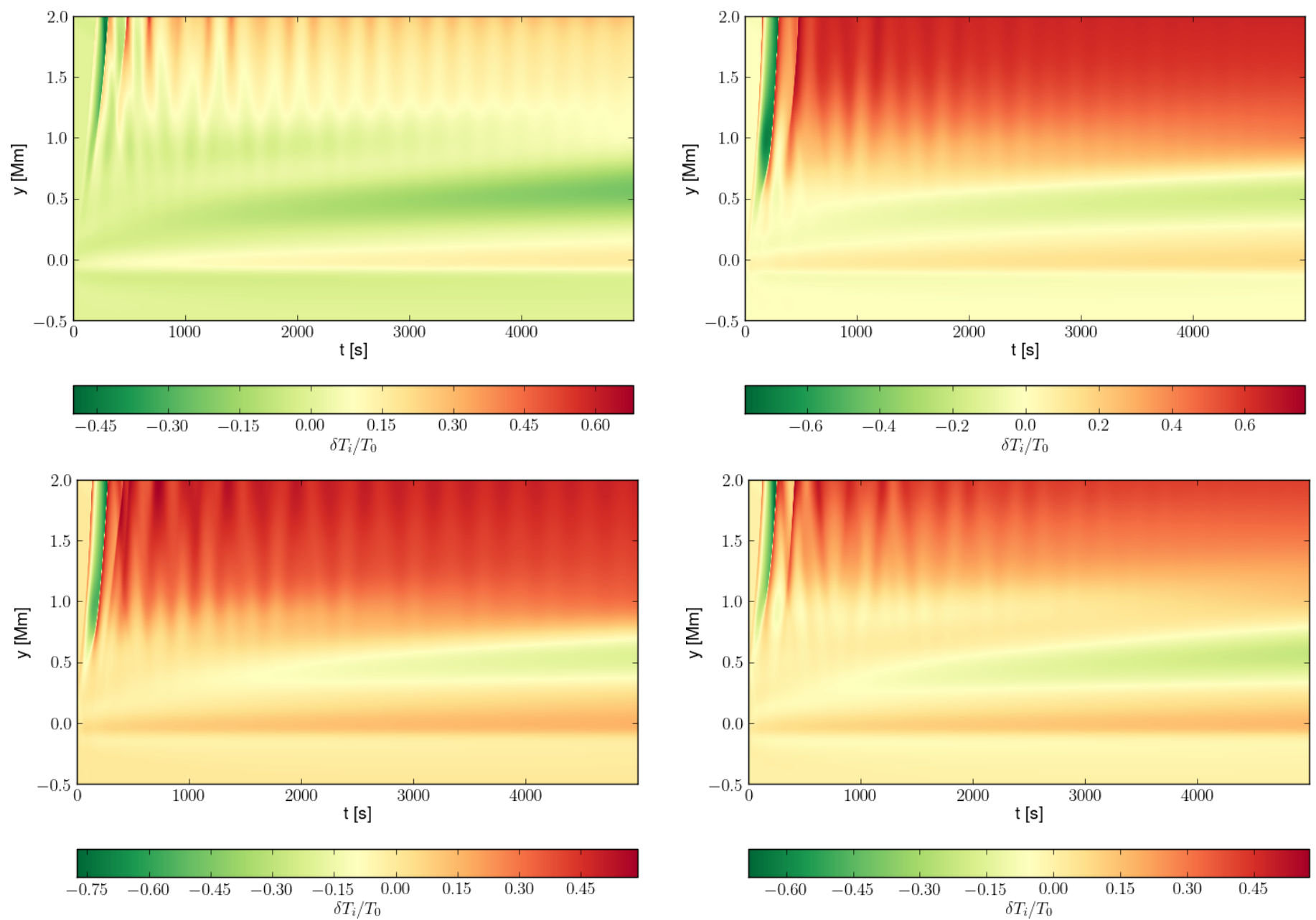

Fig. 6. Time-distance plots for $\delta T_{i} / T$ for the pulse with amplitude $A=2 \mathrm{~km} \mathrm{~s}^{-1}$ and width $w=0.1 \mathrm{Mm}$ (top-left) and $w=0.25 \mathrm{Mm}$ (top-right), launched from $y=y_{0}=0 \mathrm{Mm}$, and for $w=0.3 \mathrm{Mm}$ launched from $y_{0}=0.25 \mathrm{Mm}$ (bottom-left) and $y_{0}=0.5 \mathrm{Mm}$ (bottom-right).

Here, $y_{1}=1.9 \mathrm{Mm}$. The higher value of the pulse amplitude $|A|$ results in a more significant heating of the photosphere and chromosphere (top). Similarly, a higher magnitude of the pulse width $w$ leads to a higher value of $H$ (middle). Finally, increasing the pulse launching height $y_{0}$ corresponds to less heating below the transition region with a fall-off of $H\left(y_{0}\right)$ resulting from the pressure scale-height (bottom). For a larger value of $y_{0}$, the distance to the transition region covers fewer pressure scale-heights and the signal has less chance to increase its amplitude, and, consequently, to generate stronger shocks with less thermal energy release. The value of $H=1$ would correspond to an average relative temperature increase of the photosphere and chromosphere by $100 \%$. However, we do not reach such a value in our results since the amplitude of the pulse is too small to induce such strong heating.

\subsubsection{Outflow velocities and Fourier power spectrum}

Figure 8 shows the maximum ion outflow velocity, $\max \left(\mathrm{V}_{\mathrm{iy}}\right)$, for different pulse parameters. Similarly as in Fig. 7, a larger absolute amplitude (top) and width of the pulse (middle) results in higher value of the maximum outflow velocity $\max \left(\mathrm{V}_{\mathrm{iy}}\right)$. As we increase the launching altitude of the initial pulse, $y_{0}$, the velocity naturally decreases (bottom).
Figure 9 presents Fourier power spectrum for wave period $P$ vs height. The initial pulse has a Gaussian spectrum of wave number $k$ which results in a spectrum of period $P$. The steepening of the magnetoacoustic waves results from the growing wave amplitude with height. Hence, waves with shorter wavelengths and wave periods are present for higher $y$-values in their Fourier spectra. For the pulse launched from $y=y_{0}=0 \mathrm{Mm}$ (top) the main wave period of the downward propagating waves becomes approximately the same for all values of $y<0 \mathrm{Mm}$ and it is equal to about $250 \mathrm{~s}$. Higher up, however, the period $P$ decays with increasing $y$, and attains values close to $200 \mathrm{~s}$. As a result of the cut-off only short period waves can propagate upwards while long period waves become evanescent. Hence, the relative contribution of long $P$ waves weakens with increasing $y$. Notice that some of our data fits the observational findings of Wiśniewska et al. (2016), represented by diamonds over-plotted on the power spectra, and Kayshap et al. (2018), denoted by dots. The agreement of the theory with the observational data indicates that the results can be used to determine the background structure of the solar atmosphere and confirms that wave generation by the solar granulation in the partially ionised plasma dominates the behaviour of the waves. For a pulse at the bottom of the photosphere $\left(y=y_{0}=0 \mathrm{Mm}\right.$, Fig. 9 (top)), a jump in the dominant wave period is observed close to the height $y=1.5 \mathrm{Mm}$, which reaches a magnitude of $300 \mathrm{~s}$. The signal 

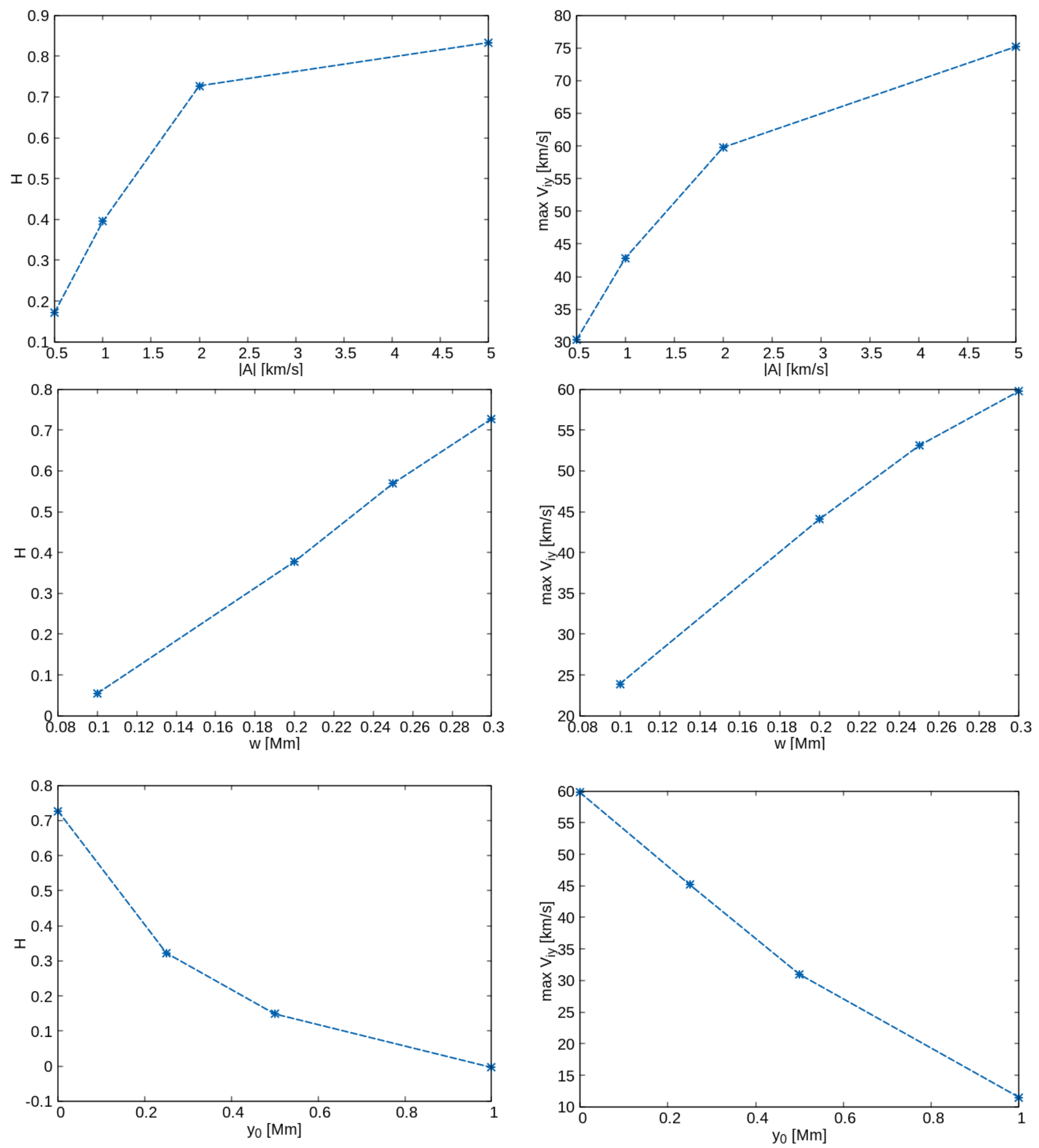

Fig. 7. Relative perturbed temperature averaged over time and height, $H$, vs: $|A|$, for $y_{0}=0 \mathrm{Mm}$ and $w=0.3 \mathrm{Mm}$ (top); $w$ for $A=2 \mathrm{~km} \mathrm{~s}^{-1}$ and $y_{0}=0 \mathrm{Mm}$ (middle); $y_{0}$ for $A=2 \mathrm{~km} \mathrm{~s}^{-1}$ and $w=0.3 \mathrm{Mm}$ (bottom).

launched from the bottom of the photosphere with the main pe$\operatorname{riod} P=250 \mathrm{~s}$ thus reaches the corona with $P=300 \mathrm{~s}$. The wave periods in the photosphere are lower than $P_{\mathrm{ac}}$ in this region, since $\max P_{\mathrm{ac}}=240 \mathrm{~s}$ at $y=0.5 \mathrm{Mm}$, which means that

Fig. 8. Maximum value of $V_{\text {iy }}$ vs: $|A|$, for $y_{0}=0 \mathrm{Mm}$ and $w=0.3 \mathrm{Mm}$ (top); $w$ for $A=2 \mathrm{~km} \mathrm{~s}^{-1}$ and $y_{0}=0 \mathrm{Mm}$ (middle); $y_{0}$ for $A=2 \mathrm{~km} \mathrm{~s}^{-1}$ and $w=0.3 \mathrm{Mm}$ (bottom).

magnetoacoustic waves are evanescent. However, in the corona above $y=2.3 \mathrm{Mm}$ the acoustic cut-off period reaches values larger than $P=300 \mathrm{~s}$. This is, however, not the case for a pulse launched at somewhat higher, in the middle of the photosphere, i.e. for $y=y_{0}=0.25 \mathrm{Mm}$ (see Fig. 9 (bottom)). As a mat- 

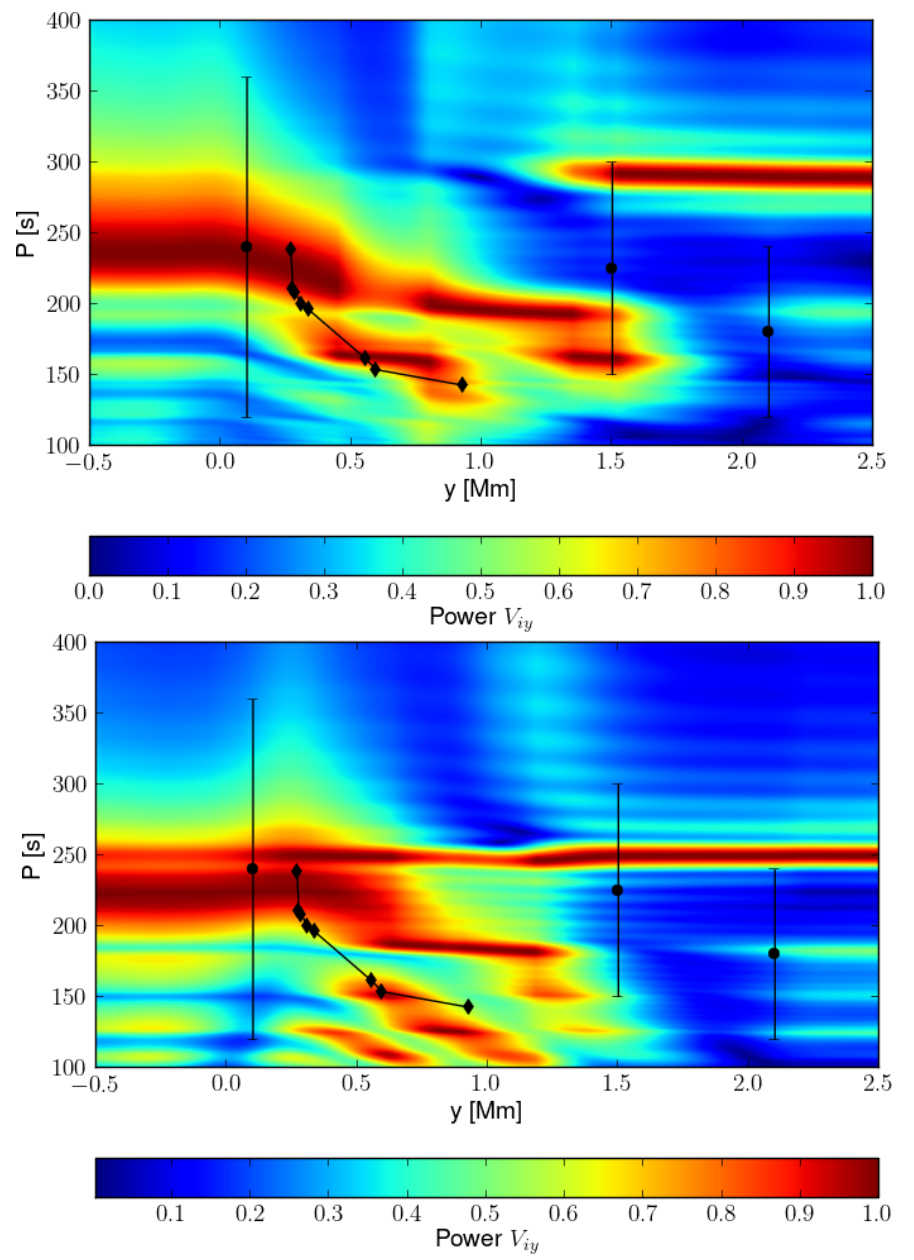

Fig. 9. Fourier period $P$ for $V_{\text {iy }}$ versus height, in the case of the initial pulse with amplitude $A=0.5 \mathrm{~km} \mathrm{~s}^{-1}$, width $w=0.3 \mathrm{Mm}$, and launched from $y=y_{0}=0 \mathrm{Mm}$ (top) and $y=y_{0}=0.25 \mathrm{Mm}$ (bottom). The observational data of Wiśniewska et al. (2016) and Kayshap et al. (2018) are represented by diamonds and dots with the vertical error bars, respectively.

ter of fact,in this case the layers below the photosphere $(y<0)$ oscillate with a dominant wave period of $225 \mathrm{~s}$. In the photosphere too this wave period is dominant and this time it increases slightly with height in the photosphere. Moreover, in this case there is a second dominant period, viz. $250 \mathrm{~s}$. In fact, this wave period is also the dominant one in the upper chromosphere and low corona in this case.

\section{Summary and Conclusions}

In this paper we considered two-fluid ion magnetoacoustic and neutral acoustic waves that are initially (at $t=0 \mathrm{~s}$ ) excited by a pulse in a vertical component of the ion and neutral velocities. The full set of two-fluid equations is solved by the JOANNA code (Wójcik et al. 2020b). The results of the numerical simulations presented in this paper can be summarised as follows.

The triggered magnetoacoustic waves transform into shock waves and through ion-neutral collisions they convert their energies into thermal energy contributing to heating of the chromospheric plasma. Increasing the amplitude of the pulse leads to a significant heating of the chromosphere, which for the highest absolute value of the amplitude considered, $A=-5 \mathrm{~km} \mathrm{~s}^{-1}$, is clearly seen to occur in the upper part of the chromosphere, above the level $y=1 \mathrm{Mm}$. Moreover, the heating of the chromosphere is correlated with a velocity drift which increases with height up to $y=2 \mathrm{Mm}$. This means that the chromosphere is heated by the ion-neutral collisions. The parametric studies we performed for various widths of the pulse show that a wider pulse corresponds to more plasma heating spread over the whole chromosphere. On the other hand, the investigation of quasi-periodic MHD waves by Nakariakov et al. (2005) showed that a wider pulse is less effective in generating quasi-periodic wave trains. The problem of impulsively generated wave trains was also studied by Yu et al. (2017) who concluded that the diversity of group speed characteristics has an impact on the temporal evolution of impulsively generated wave trains. A pulse launched from a higher level (with the same amplitude), on the other hand, results in less heating as the amplitude of upwardly propagating signal cannot grow that much with height before reaching the transition region. Both the plasma temperature and maximum ion velocity increase with increasing pulse amplitude and width, while the opposite trend is seen for higher values of $y_{0}$, i.e. for launching the pulse higher up. The pulses considered in this paper result in shocks which lead to a release of thermal energy which in turn helps to sustain the chromosphere. Another conclusion is that even a single pulse can drive vertical plasma outflows which higher up can result in (contributions to) the origin of the solar wind.

\section{Acknowledgments}

The authors express their gratitude to the reviewer for his/her stimulating comments and suggestions. The JOANNA code was written by Dr. Dariusz Wójcik. This work was done within the framework of the project from the National Science Centre (NCN) grant nos. 2017/25/B/ST9/00506 and 2020/37/B/ST9/00184. Numerical simulations were performed on the LUNAR cluster at the Institute of Mathematics of University of M. Curie-Skłodowska, Lublin, Poland. SP is supported by the project EUHFORIA 2.0 which has received funding from the European Union's Horizon 2020 research and innovation programme under grant agreement No 870405, and by the projects C14/19/089 (C1 project Internal Funds KU Leuven), G.0D07.19N (FWO-Vlaanderen), SIDC Data Exploitation (ESA Prodex-12), and the Belspo projects BR/165/A2/CCSOM and B2/191/P1/SWiM.

\section{References}

Arber, T. D., Brady, C. S., \& Shelyag, S. 2016, ApJ, 817, 94

Avrett, E. H. \& Loeser, R. 2008, ApJS, 175, 229

Ballester, J. L., Alexeev, I., Collados, M., et al. 2018, Space Sci. Rev., 214, 58 Biermann, L. 1946, NW, 33, 118

Biermann, L. 1951, ZAp, 29, 274

Braginskii, S. I. 1965, Reviews of Plasma Physics, 1, 205

Carlsson, M. \& Stein, R. F. 1995, ApJ, 440, L29

Carlsson, M. \& Stein, R. F. 2002, ApJ, 572, 626

De Pontieu, B., Martens, P. C. H., \& Hudson, H. S. 2001, ApJ, 558, 859

De Pontieu, B., McIntosh, S. W., Carlsson, M., et al. 2007, Science, 318, 1574

Duckenfield, T. J., Kolotkov, D. Y., \& Nakariakov, V. M. 2021, A\&A, 646, A155 Erdélyi \& James. 2004, A\&A, 427, 1055

Falco, Puglisi, G., Guglielmino, S. L., et al. 2017, A\&A, 605, A87

Goodman, M. L. 2011, ApJ, 735, 45

Hollweg, J. V., Jackson, S., \& Galloway, D. 1982, Sol. Phys., 75, 35

Horace, L. 1909, Proceedings of the London Mathematical Society, s2-7, 122

Kayshap, P., Banerjee, D., \& Srivastava, A. K. 2015, Solar Physics, 290, 2889

Kayshap, P., Murawski, K., Srivastava, A. K., Musielak, Z. E., \& Dwivedi, B. N. 2018, MNRAS, 479, 5512

Khodachenko, Arber, T. D., Rucker, H. O., \& Hanslmeier, A. 2004, A\&A, 422, 1073 
Khomenko, E., Collados, M., Díaz, A., \& Vitas, N. 2014a, Physics of Plasmas, 21, 092901

Khomenko, E., Collados, M., Díaz, A., \& Vitas, N. 2014b, Physics of Plasmas, 21, 092901

Kolotkov, D. Y., Duckenfield, T. J., \& Nakariakov, V. M. 2020, A\&A, 644, A33

Kolotkov, D. Y., Nakariakov, V. M., \& Zavershinskii, D. I. 2019, A\&A, 628, A133

Kudoh, T. \& Shibata, K. 1999, ApJ, 514, 493

Kuźma, B., Murawski, K., Musielak, Z. E., Poedts, S., \& Wójcik, D. 2021, arXiv e-prints, arXiv:2105.09882

Kuźma, B., Wójcik, D., \& Murawski, K. 2019, ApJ, 878, 81

Leake, J. E., Arber, T. D., \& Khodachenko, M. L. 2005, A\&A, 442, 1091

Leake, J. E., DeVore, C. R., Thayer, J. P., et al. 2014, Space Sci. Rev., 184, 107

Maneva, Y. G., Alvarez Laguna, A., Lani, A., \& Poedts, S. 2017, ApJ, 836, 197

Marsch, E. 2006, Living Reviews in Solar Physics, 3, 1

Matsumoto, T. \& Suzuki, T. K. 2012, ApJ, 749, 8

Meier, E. T. \& Shumlak, U. 2012, Physics of Plasmas, 19, 072508

Musielak, Z. E., Musielak, D. E., \& Mobashi, H. 2006, Phys. Rev. E, 73, 036612

Nakariakov, V. M., Afanasyev, A. N., Kumar, S., \& Moon, Y. J. 2017, ApJ, 849,

Nakariakov, V. M., Pascoe, D. J., \& Arber, T. D. 2005, Space Sci. Rev., 121, 115

Nakariakov, V. M. \& Verwichte, E. 2005, Living Reviews in Solar Physics, 2, 3

Narain, U. \& Ulmschneider, P. 1996, Space Science Reviews, 75, 453

Ofman, L. 2005, Space Sci. Rev., 120, 67

Oliver, R., Soler, R., Terradas, J., \& Zaqarashvili, T. V. 2016, ApJ, 818, 128

Osterbrock, D. E. 1961, ApJ, 134, 347

Parker, E. N. 1958, ApJ, 128, 664

Parker, E. N. 1965, Space Sci. Rev., 4, 666

Piddington, J. H. 1956, MNRAS, 116, 314

Popescu Braileanu, B., Lukin, V. S., Khomenko, E., \& de Vicente, Á. 2019, A\&A, 627, A25

Prasad, A., Srivastava, A. K., \& Wang, T. J. 2021, Sol. Phys., 296, 20

Schwarzschild, M. 1948, The Astrophysical Journal, 107, 1

Shelyag, S., Khomenko, E., Przybylski, D., Vitas, N., \& de Vicente, A. 2016, in AGU Fall Meeting Abstracts, SH21E-2565

Snow, B. \& Hillier, A. 2021, A\&A, 645, A81

Soler, R., Terradas, J., Oliver, R., \& Ballester, J. L. 2017, ApJ, 840, 20

Tian, H., Harra, L., Baker, D., Brooks, D. H., \& Xia, L. 2021, Sol. Phys., 296,

Tu, C.-Y. 1987, Sol. Phys., 109, 149

Tu, C.-Y., Zhou, C., Marsch, E., et al. 2005, Science, 308, 519

Tu, J. \& Song, P. 2013, ApJ, 777, 53

Ulmschneider, P. \& Musielak, Z. 2003, in Astronomical Society of the Pacific Conference Series, Vol. 286, Current Theoretical Models and Future High Resolution Solar Observations: Preparing for ATST, ed. A. A. Pevtsov \& H. Uitenbroek, 363

van der Holst, B., Sokolov, I. V., Meng, X., et al. 2014, ApJ, 782, 81

Van Doorsselaere, T., Srivastava, A. K., Antolin, P., et al. 2020, Space Sci. Rev. 216,140

Vranjes, J. \& Krstic, P. S. 2013, A\&A, 554, A22

Wiśniewska, A., Musielak, Z. E., Staiger, J., \& Roth, M. 2016, ApJ, 819, L23

Wójcik, D., Kuźma, B., Murawski, K., \& Musielak, Z. E. 2020a, A\&A, 635, A28

Wójcik, D., Kuźma, B., Murawski, K., \& Musielak, Z. E. 2020b, A\&A, 635, A28

Wójcik, D., Kuźma, B., Murawski, K., \& Srivastava, A. K. 2019, ApJ, 884, 127

Yu, H., Li, B., Chen, S.-X., Xiong, M., \& Guo, M.-Z. 2017, ApJ, 836, 1

Zaqarashvili, T. V., Khodachenko, M. L., \& Rucker, H. O. 2011a, A\&A, 534, A93

Zaqarashvili, T. V., Khodachenko, M. L., \& Rucker, H. O. 2011b, A\&A, 529, A82

Zaqarashvili, T. V., Khodachenko, M. L., \& Soler, R. 2013, A\&A, 549, A113

Zhang, F., Poedts, S., Lani, A., KUŹMA, B., \& Murawski, K. 2020, arXiv eprints, arXiv:2011.13469 